\title{
ALTERNATIVE THEORETICAL FRAMEWORKS FOR HYBRID SANDWICH PANEL WITH INTERMEDIATE LAYER
}

\author{
Jauhar Fajrin ${ }^{1}$
}

\begin{abstract}
A concept of hybrid sandwich panel with intermediate layer has been introduced previously that capable of improving load carrying capacity of composite sandwich panel. While the previous work employed numerical and experimental investigation, the current paper more focuses on developing the theoretical frameworks of the hybrid sandwich panel by using analytical model. The vocal point of the concept is introducing a new layer in between the skin and the core of a standard sandwich panel structure to form a hybrid structure. The paper begins with elaborating the basic concept of sandwich panel which includes the elastic and shear stresses in sandwich panel and followed by the deflection of sandwich panel. The next stage comprehensively highlights the theoretical concept of the hybrid sandwich panel. It was clearly shown through the developed theoretical frameworks that the incorporation of intermediate layer can substantially increase the flexural rigidity and stiffnesses which finally enhances the load carrying capacity of the new developed sandwich panel.
\end{abstract}

Keywords : sandwich panel, hybrid concept, intermediate layer, analytical model.

\section{INTRODUCTION}

Composite sandwich panel has been widely used in manufacturing industry such as aerospace, marine and automotive. It has now transitioned to become a viable choice to other application field such as civil infrastructure, particularly for lightweight applications. High strength to weight ratio is the most recognized advantage of composite sandwich panels that positioned them in the first list of lightweight material choices. The conservative form of sandwich structure consists of two thin stiff and strong face layers which are separated by a lightweight core material. The face sheets are bonded to the core using structural adhesive to obtain a load transfer between the components. The face sheets will act together to carry external bending moment, while the primary purposeof the core is to resist shear and to stabilize the faces against buckling or wrinkling (Zenkert, 1995).

A lot of research works have been done on improving the properties of compositesandwich panel. The first category is the enhancement of face sheet materials which have been extensively investigated by many researchers. The most important attempt for this category was the introduction of fiber composites skin which has major impact on the use of composite sandwich panel. In the second category, extensive works have been carried out on improving the properties of core materials. In the third category, few studies have also been carried out on introducing new element to improve the properties of composite sandwich panel. Including in the third category was a concept of hybrid sandwich panel with intermediate layer which was introduced byMamalis et al. (2008). The new concept has attracted a particular attention as it has significantly enhanced the

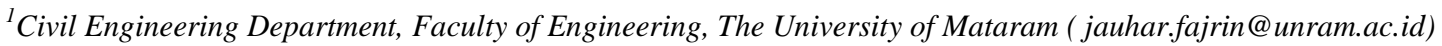


behaviour of composite sandwich panel by a relatively simple method. A finite element approach was employed on their study for assessingseveral possible cases to find optimum material configurations in term of price. While the work of Mamalis et al. (2008) employed numerical and experimental investigation, the study reported in this paper more focuses on developing theoretical frameworks as an alternative to the Mamalis' concept of the hybrid sandwich panel by using analytical model. The concept has been extensively examined previously using statistical design of experiment approaches; simple comparative experiment method (Fajrin et al., 2011) and single factor experiment method (Fajrin et al., 2011). A significance analysis of the flexural behaviour of the hybrid sandwich panel was also reported by Fajrin et al.(2013).

\section{BASIC CONCEPT OF SANDWICH PANEL}

A sandwich structure typically consists of three elements; face sheets, core and adhesive. Every part of the panel has a specific function to enable the panel work as a unit. A sandwich beam of the same width and weight as a solid beam has a considerable higher stiffness due to its higher moment of inertia (DIAB, 2009). The adhesive has an important role to ensure that faces and the core are fully bonded out but it is often neglected as a part the sandwich panel. The theoretical frameworks developed here were adapted from few selected literatures (Allen, 1969; Zenkert, 1995; DIAB, 2001 and Deshpande, 2002) but is largely based on the work of Deshpande (2002).

Consider a sandwich beam with a uniform width (b) and face sheets of thickness t perfectly bonded to a foam core with the thickness of $\mathrm{c}$. The beam is loaded in 3-point bending as shown in Figure 2.1 with a span $\mathrm{L}$. Let $\mathrm{E}_{\mathrm{f}}$ is the Young's moduli of faces and $\mathrm{E}_{\mathrm{c}}$ is the Young's moduli of the core. The stress and deflections in a beam of this may be obtained by simple beam bending theory. In this initial stage of analysis the theory is based upon the assumption that cross-sections are plane and perpendicular to the longitudinal axis of the unloaded beam remain so when bending takes place. This assumption leads to the well understood relation between the bending moment and the curvature $(1 / \mathrm{R})$. TheEI in this relation is the flexural rigidity.

$$
\frac{\mathrm{M}}{\mathrm{EI}}=-\frac{1}{\mathrm{R}}
$$

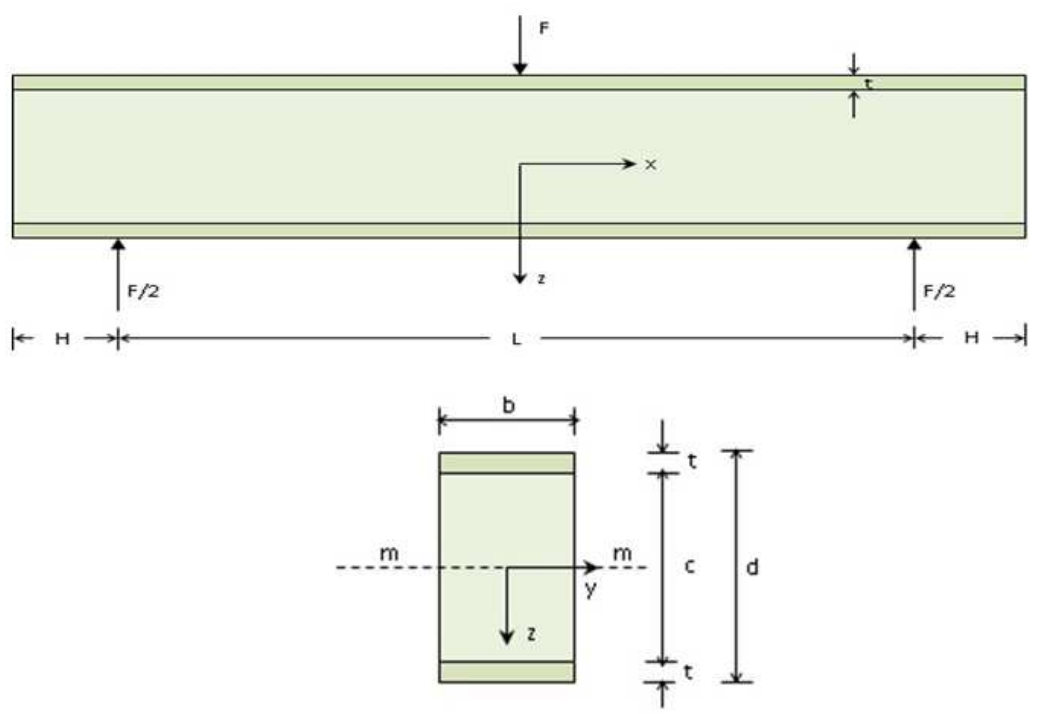

Figure 2.1. Long and cross sections of sandwich panel loaded in 3-point bending 
The equivalent flexural rigidity (EI) eq ofa homogeneous sandwich beamconsists of the sum of the rigidities of the faces and core measured about the neutral axis, $\mathrm{m}-\mathrm{m}$, of the entire sections.

$$
\begin{aligned}
& (E I)_{\text {core }}=E_{c} \cdot \frac{1}{12} b c^{3}=\frac{1}{12} E_{c} b c^{3} \\
& (E I)_{\text {faces }}=E_{f} \cdot I_{f}, \text { about } m-m
\end{aligned}
$$

Using parallel axis theorem, $\mathrm{I}_{\text {faces }}$ can be calculated as follows.

$$
\begin{aligned}
& I_{\text {faces }}=2\left[I_{\text {faces }}+A\left(\frac{d}{2}\right)^{2}\right] \\
& I_{\text {faces }}=\frac{1}{6} b t^{3}+\frac{b t^{2}}{2}
\end{aligned}
$$

So that,

$$
(E I)_{\text {faces }}=\frac{1}{6} E_{f} b t^{3}+\frac{1}{2} E_{f} b t d^{2}
$$

Thus,

$$
\begin{aligned}
& (E I)_{\text {eq }}=(E I)_{\text {faces }}+(E I)_{\text {core }} \\
& (E I)_{\text {eq }}=E_{\mathrm{f}} \frac{\mathrm{bt}^{3}}{6}+E_{\mathrm{f}} \frac{\mathrm{btd}^{2}}{2}+\mathrm{E}_{\mathrm{c}} \frac{\mathrm{bc}^{3}}{12}
\end{aligned}
$$

In most practical sandwich panel, the faces are usually thin compared with the core and the first term of Equation 8 is therefore quite small and is less than $1 \%$ of the second value when:

$$
\frac{\mathrm{d}}{\mathrm{t}}>5.77
$$

The core usually has a much lower modulus than that of the face and the third term in Equation 8 is less than $1 \%$ if:

$$
\frac{E_{f}}{E_{c}} \frac{t^{2}}{c^{3}}>16.7
$$

If the conditions in Equation 9 and 10 are satisfied, then the flexural rigidity of sandwich panel may reduce to:

$$
(\mathrm{EI})_{\mathrm{eq}} \approx \mathrm{E}_{\mathrm{f}} \frac{\mathrm{btd}^{2}}{2}
$$

As indicated earlier, sandwich panel has a high stiffness because of its high moment of inertia. The stiffness of the above sandwich beam is given by:

$$
\mathrm{k}=\frac{\mathrm{F}}{\delta}
$$

Where, $\mathrm{k}$ is the stiffness, $\mathrm{F}$ is the applied force and $\delta$ is the displacement which in this case is the deflection. The deflection of a homogeneous beam under 3-point bending load is,

$$
\delta=\frac{\mathrm{FL}^{3}}{48 \mathrm{EI}}
$$


In the case of sandwich panel, this equation can be modified as:

$$
\delta=\frac{\mathrm{FL}^{3}}{48(\mathrm{EI})_{\mathrm{eq}}}
$$

Hence, by including Equation 14 into Equation 12, a stiffness of a sandwich panel can be obtained as follows.

$$
\mathrm{k}=\frac{48(\mathrm{EI})_{\mathrm{eq}}}{\mathrm{L}^{3}}
$$

Based on Equation 15, it can be seen that the higher flexural rigidity(EI) $)_{\mathrm{eq}}$, the higher beam stiffness. Using the sandwich concept the flexural rigidity and stiffness of a beam can be substantially enhanced, without much increase in weight.

\subsection{Elastic Stresses in Sandwich Panel}

This part was mostly adapted from the work of Deshpande (2002). The stress in the faces and core can be obtained by using ordinary beam theory. As the sections remain plane and perpendicular to the longitudinal axis, the longitudinal strain at a point of $\mathrm{z}$ is given by,

$$
\varepsilon_{1}=\frac{z}{R}
$$

The value of $\mathrm{R}$ can be obtained by rewriting Equation 1 and inserts the result into the Equation 16, which results in:

$$
\varepsilon_{1}=\frac{\mathrm{Mz}}{(\mathrm{EI})_{\mathrm{eq}}}
$$

The obtained strain form the Equation 17 may be multiplied by the appropriate modulus of elasticity to give the bending stress at level z. For example, the stresses in the faces and core are given by:

$$
\begin{gathered}
\sigma_{\mathrm{f}}=\frac{\mathrm{Mz}}{(\mathrm{EI})_{\mathrm{eq}}} \mathrm{E}_{\mathrm{f}} \\
\text { for }\left[\frac{\mathrm{c}}{2} \leq \mathrm{z} \leq \frac{\mathrm{c}+\mathrm{t}}{2}\right] ;\left[-\frac{\mathrm{c}+\mathrm{t}}{2} \leq \mathrm{z} \leq-\frac{\mathrm{c}}{2}\right] \\
\sigma_{\mathrm{c}}=\frac{\mathrm{Mz}}{(\mathrm{EI})_{\mathrm{eq}}} \mathrm{E}_{\mathrm{c}} \\
\text { for }\left[-\frac{\mathrm{c}}{2} \leq \mathrm{z} \leq \frac{\mathrm{c}}{2}\right]
\end{gathered}
$$

Thus, the maximum face and core stresses are achieved with $\mathrm{z}= \pm(\mathrm{c}+\mathrm{t}) / 2$ and $\mathrm{z}= \pm \mathrm{c} / 2$. Hence, the stresses vary linearly within each material constituent, but there is a jump in the stress at the face and core interface. 


\subsection{Shear Stresses in Sandwich Panel}

The shear $\operatorname{stress}(\tau)$ in a homogeneous beam, as shown in Figure 2.2, at a depth $\mathrm{z}$ is defined by Equation 20.

$$
\tau=\frac{Q}{b I} A_{c} z_{c}
$$

Where; $Q$ is the shear force, $A_{c}$ is the area of cut off portion and $A_{c} z_{c}$ is the first moment of area of the cut-off portion about thecentroidal axis.

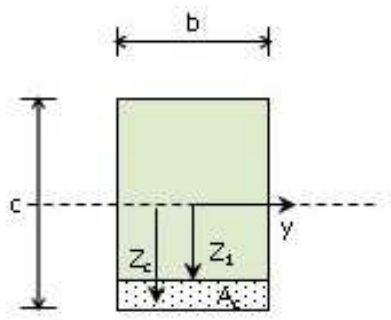

Figure 2.2. The cross section in shear analysis for homogeneous beam

In the case of a sandwich beam, this equation is modified to consider the contribution of the moduli of elasticity of the different elements of the cross-section.

$$
\tau=\frac{\mathrm{Q}}{\mathrm{b}(\mathrm{EI})_{\mathrm{eq}}} \sum \mathrm{A}_{\mathrm{c}} \mathrm{z}_{\mathrm{c}} \mathrm{E}
$$

The summation $\sum$ is done for all parts of the section for whichz $<z_{1}$. For instance, to determine the shear stress at level $\mathrm{z}$ in the core of the sandwich, as shown in Figure 2.3, the procedure is as follows.

$$
\begin{aligned}
& \sum A_{c} z_{c} E=E_{f} \cdot A_{f} \cdot \frac{d}{2}+E_{c} \cdot A_{c} \cdot\left[\frac{1}{2}\left(\frac{c}{2}-z\right)+z\right] \\
& \sum A_{c} b_{c} E=\frac{E_{f} b t d}{2}+\frac{E_{c} \cdot b}{2}\left(\frac{c^{2}}{4}-z^{2}\right)
\end{aligned}
$$

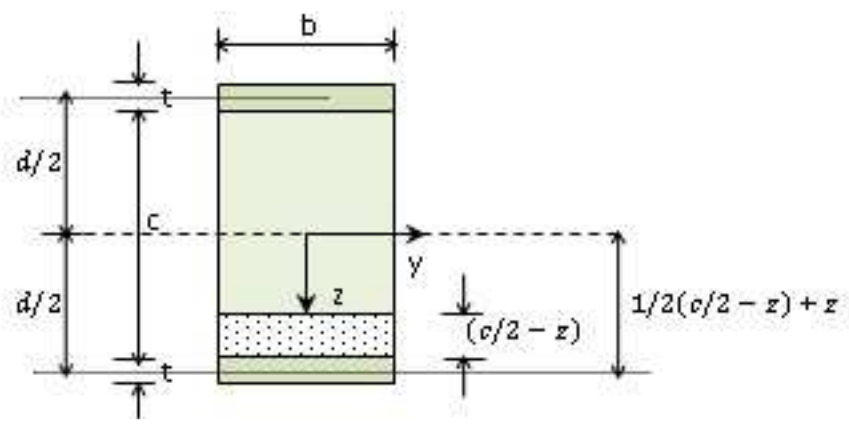

Figure 2.3. Sketch of sandwich beam cross section for shear analysis

Thus, as the shear stress in the core is defined as per Equation 21, then:

$$
\tau=\frac{\mathrm{Q}}{\mathrm{b}(\mathrm{EI})_{\mathrm{eq}}}\left[\frac{\mathrm{E}_{\mathrm{f}} \mathrm{btd}}{2}+\frac{\mathrm{E}_{\mathrm{c}} \cdot \mathrm{b}}{2}\left(\frac{\mathrm{c}^{2}}{4}-\mathrm{z}^{2}\right)\right]
$$


Eliminating the factor of $b$, gives the final equation to obtain shear stress in the core:

$$
\tau=\frac{\mathrm{Q}}{(\mathrm{EI})_{\mathrm{eq}}}\left[\frac{\mathrm{E}_{\mathrm{f}} \mathrm{td}}{2}+\frac{\mathrm{E}_{\mathrm{c}}}{2}\left(\frac{\mathrm{c}^{2}}{4}-\mathrm{z}^{2}\right)\right]
$$

A similar expression may be obtained the shear stress in the faces and the complete of shear stress distribution across the depth of the sandwich is illustrated in Figure 2.4(A). For a normal sandwich panel, $E_{c}<<E_{f}$, so the second term in the Equation 25 can be neglected and reduced to:

$$
\tau=\frac{\mathrm{Q}}{(\mathrm{EI}) \mathrm{eq}} \cdot \frac{\mathrm{E}_{\mathrm{f}} \mathrm{td}}{2}
$$

Considering Equation 11 where approximate $\left.(\mathrm{EI})_{\mathrm{eq}}=\mathrm{E}_{\mathrm{f}} \mathrm{btd}{ }^{2}\right) / 2$, the shear stress in the core can be simplified as follows.

$$
\begin{aligned}
& \tau=\frac{Q}{\left.E_{\mathrm{f}} \mathrm{btd}^{2}\right) / 2} \cdot \frac{\mathrm{E}_{\mathrm{f}} \mathrm{td}}{2} \\
& \tau=\frac{\mathrm{Q}}{\mathrm{bd}}
\end{aligned}
$$

The corresponding shear stress distribution in the sandwich beam is shown in Figure 2.4(B).

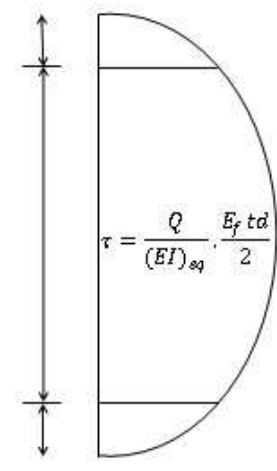

(A)

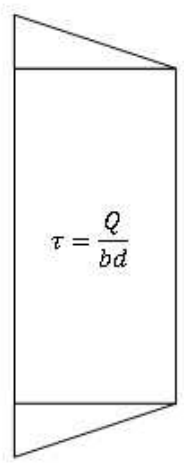

(B)

Figure 2.4. (A) The shear stress in the faces and the complete shear stress distribution across the depth of the sandwich. (B) The corresponding simplified shear stress. (Deshpande, 2002).

\subsection{Deflection of a Sandwich Panel}

The deflection of a homogeneous material due to shear is often neglected. For a sandwich panel, however, the core material is usually not rigid in shear and thus the deflection is not negligible in most cases (Zenkert, 1995). As it has been presented in pervious equations, the deflection of sandwich panel can be obtained by adoption of the previous basic equation of beam deflection. Two previous subsequent equations, Equation 13 and Equation 14, showed how the basic beam deflection equation has been modified for a sandwich panel beam. Equation 15 shows that the increasing the separation of the face sheets increases the flexural rigidity and stiffness of a sandwich beam. While separation should be increased as much as possible, it may induce a shear mode of deformation that commonly neglected in ordinary beam analysis.

Recalling back at the assumption made for the ordinary beam bending theory, it was assumed that cross-sections that are plane and perpendicular to the longitudinal axis of the unloaded beam 
remain so when bending takes place. As it seen in Figure 2.5, the cross-section aa, bb, cc and dd has been slightly rotated but remain perpendicular to the longitudinal axis of the deflected beam. The upper part of the beam is under compression and the lower part is under tension (DIAB, 2001).
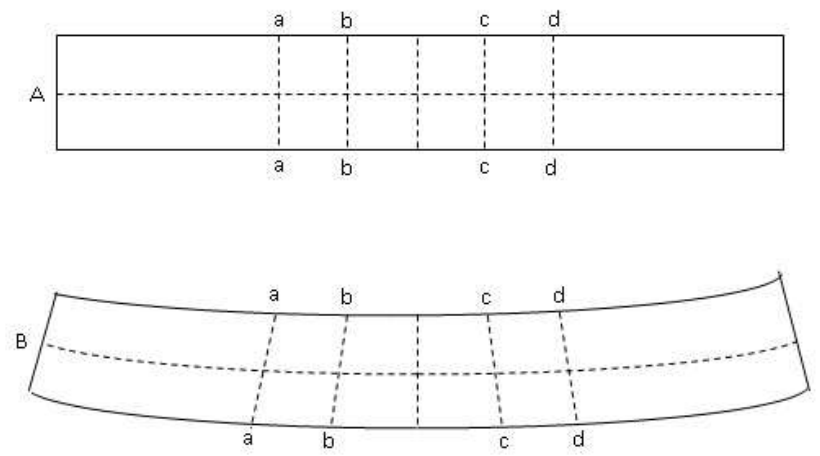

Figure 2.5. Basic assumption in ordinary beam theory

The shear stress in the core at any section has been defined by the Equation 27. This equation is relatedtothe following shear strain.

$$
\gamma=\frac{\mathrm{Q}}{\mathrm{G}_{\mathrm{c}} \mathrm{bd}}
$$

The above equation was provided by the following process.

$$
\begin{aligned}
& \mathrm{G}_{\mathrm{c}}=\frac{\tau}{\gamma} \\
& \gamma=\frac{\tau}{\mathrm{G}_{\mathrm{c}}} \\
& \gamma=\frac{1}{\mathrm{G}_{\mathrm{c}}} \cdot \frac{\mathrm{Q}}{\mathrm{bd}}
\end{aligned}
$$

Where $G_{c}$ is the shear modulus of the core material. This shear strain leads to a new kind of deformation as shown in Figure 2.6.

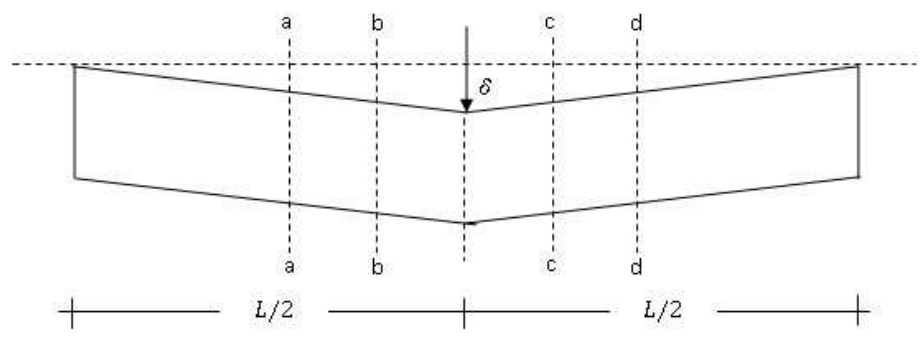

Figure 2.6.New type of deformation due to core shear.

As shown in the Figure 2.6, the points a, b, c, and d which lie on the centre line of the faces are displaced vertically. The points do not move horizontally. The deflection of the loading point due to this deformation mode is given by.

$$
\frac{\delta}{\mathrm{L} / 2}=\gamma=\frac{\mathrm{Q}}{\mathrm{G}_{\mathrm{c}} \mathrm{bd}}
$$




$$
\delta=\frac{\mathrm{Q} \cdot \mathrm{L} / 2}{\mathrm{G}_{\mathrm{c}} \mathrm{bd}}
$$

Under 3-point bending load, $\mathrm{Q}$ is equal to $\mathrm{F} / 2$, then:

$$
\begin{aligned}
& \delta=\frac{F / 2 . L / 2}{\mathrm{G}_{\mathrm{c}} \mathrm{bd}} \\
& \delta=\frac{\mathrm{FL}}{4\left(\mathrm{G}_{\mathrm{c}} \mathrm{bd}\right)} \\
& \delta=\frac{\mathrm{FL}}{4(\mathrm{AG})_{\mathrm{eq}}}
\end{aligned}
$$

The total deflection at the centre point of the beam due to the bending (Equation 14) and shear deformation (Equation 36) can be obtained by a linear superposition, which gives:

$$
\delta=\frac{\mathrm{FL}^{3}}{48(\mathrm{EI})_{\mathrm{eq}}}+\frac{\mathrm{FL}}{4(\mathrm{AG})_{\mathrm{eq}}}
$$

This is a more appropriate equation for the deflection of sandwich panels and the equation for their stiffness also has to be modified. When considering the shear contribution in the deflection of sandwich panel, the stiffness of sandwich beam can be calculated based on the following equations.

$$
\mathrm{k}=\frac{\mathrm{F}}{\frac{\mathrm{FL}^{3}}{48(\mathrm{EI}) \mathrm{eq}}+\frac{\mathrm{FL}}{4(\mathrm{AG})_{\mathrm{eq}}}}
$$

By rearranging this equation, the stiffness of sandwich beam can be obtained by the following equation.

$$
\mathrm{k}=\frac{48(\mathrm{EI})_{\mathrm{eq}}}{\mathrm{L}^{3}}+\frac{4(\mathrm{AG})_{\mathrm{eq}}}{\mathrm{L}}
$$

\section{THE HYBRID SANDWICH PANEL MODEL}

The hybrid structure of the sandwich panel introduced in this paper is achieved by placing one more layer, which is called as an intermediate layer, between the core and the skins (Mamalis et al., 2008). The term hybrid arises from the fact that a new constituent has been incorporated in an ordinary sandwich panel structure which typically consists of only two elements, faces and core. By introducing this new layer the sandwich panel has now consists of three materials that is skins, intermediate layers, and the core. The analysis of the behaviour of new hybrid sandwich panel is basically carried out by taking into account the contribution of this new element. However, the basic analysis remains the same as for the ordinary sandwich panel.

Consider a sandwich beam of uniform width (b), with two identical intermediate layer of thickness $t_{i}$ perfectly bonded to the foam core of thickness $t_{c}$. The other two equal face sheets with thickness $t_{f}$ are also perfectly bonded to the intermediate layer of the sandwich panel to create a hybrid form. The beam is loaded in 3-point bending as sketched in Figure 3.1 with a span L. Let $E_{f}, E_{i}$ and $E_{c}$ be the Young's moduli of the face sheets, intermediate layer and core, respectively. The stress and deflections in this hybrid sandwich beam may be obtained in a similar way as the ordinary sandwich beam.

\section{I JURNAL REKAYASA SIPIL}




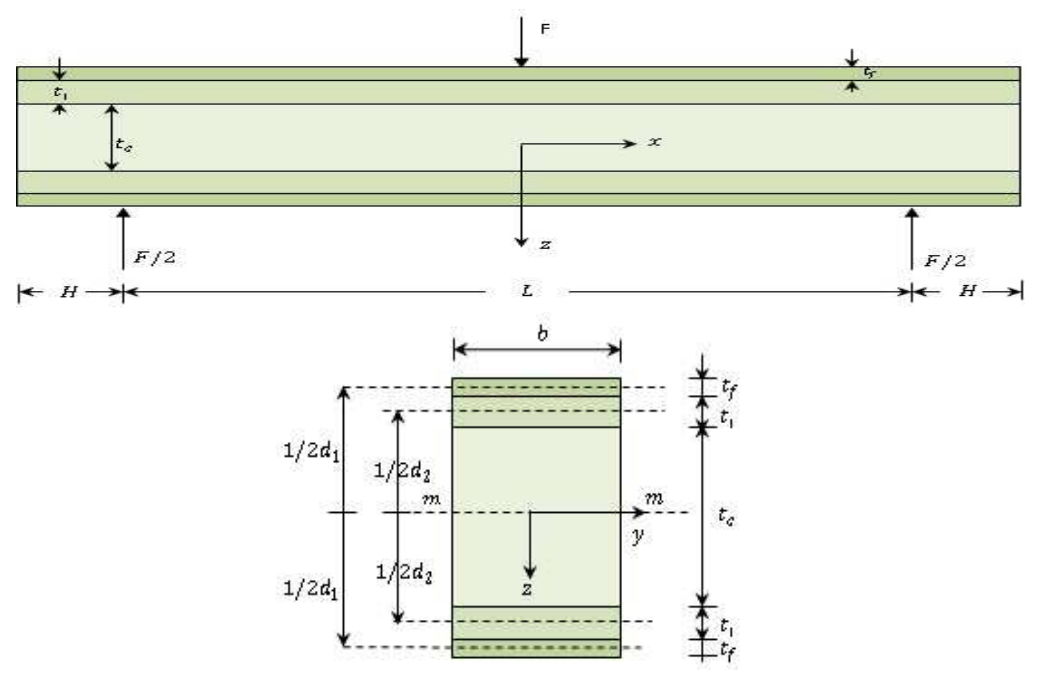

Figure 3.1. Long and cross sections of hybrid sandwich panel loaded in 3-point bending

For this hybrid sandwich beam, the equivalent flexural rigidity (EI) eq consists of the sum of the rigidities of the faces, intermediate layer and core measured about the neutral axis, $\mathrm{m}-\mathrm{m}$, of the entirely sections.

$$
\begin{aligned}
& (E I)_{\text {core }}=E_{c} \cdot \frac{1}{12} b t_{c}^{3}=\frac{1}{12} E_{c} b t_{c}^{3} \\
& (E I)_{\text {intermediate layer }}=E_{\mathrm{i}} \cdot \mathrm{I}_{\mathrm{i}} \text { about } \mathrm{m}-\mathrm{m} \\
& (\mathrm{EI})_{\text {faces }}=\mathrm{E}_{\mathrm{f}} \cdot \mathrm{I}_{\mathrm{f}} \text { about } \mathrm{m}-\mathrm{m}
\end{aligned}
$$

$\mathrm{I}_{\text {faces }}$, can be calculated from the parallel axis theorem,

$$
\begin{aligned}
& I_{\text {faces }}=2\left[I_{\text {faces }}+A\left(\frac{d_{1}}{2}\right)^{2}\right] \\
& I_{\text {faces }}=2\left[\frac{1}{12} b t_{f}^{3}+\frac{b t_{f} d_{1}^{2}}{4}\right] \\
& I_{\text {faces }}=\frac{1}{6} b t_{f}^{3}+\frac{b t_{f} d_{1}^{2}}{2}
\end{aligned}
$$

So that,

$$
(E I)_{\text {faces }}=\frac{1}{6} E_{f} b t_{f}^{3}+\frac{1}{2} E_{f} b t_{f} d_{1}^{2}
$$

Using a similar way, $\mathrm{I}_{\text {intermediate layer, }}$, can also be calculated based on the parallel axis theorem,

$$
\begin{aligned}
& \mathrm{I}_{\text {intermediate layer }}=2\left[\mathrm{I}_{\text {intermediate layer }}+\mathrm{A}\left(\frac{\mathrm{d}_{2}}{2}\right)^{2}\right] \\
& \mathrm{I}_{\text {intermediate layer }}=2\left[\frac{1}{12} \mathrm{bt}_{\mathrm{i}}^{3}+\frac{\mathrm{bt}_{\mathrm{i}} \mathrm{d}_{2}^{2}}{4}\right] \\
& \mathrm{I}_{\text {intermediate layer }}=\frac{1}{6} \mathrm{bt}_{\mathrm{i}}^{3}+\frac{\mathrm{bt}_{\mathrm{i}} \mathrm{d}_{2}^{2}}{2}
\end{aligned}
$$


So that,

$$
(E I)_{\text {intermediate layer }}=\frac{1}{6} E_{i} b t_{i}^{3}+\frac{1}{2} E_{i} b t_{i} d_{2}^{2}
$$

Hence,

$$
\begin{aligned}
& (E I)_{\text {eq }}=(E I)_{\text {faces }}+(E I)_{\text {intermediate layer }}+(E I)_{\text {core }} \\
& (E I)_{\text {eq }}=\frac{1}{6} E_{\mathrm{f}} b_{f}^{3}+\frac{1}{2} E_{\mathrm{f}} b_{\mathrm{f}} \mathrm{d}_{1}^{2}+\frac{1}{6} \mathrm{E}_{\mathrm{i}} \mathrm{bt}_{\mathrm{i}}^{3}+\frac{1}{2} \mathrm{E}_{\mathrm{i}} \mathrm{bt}_{\mathrm{i}} \mathrm{d}_{2}^{2}+\frac{1}{12} \mathrm{E}_{\mathrm{c}} \mathrm{bt}_{\mathrm{c}}^{3}
\end{aligned}
$$

Or, the above equation can be simplified as follows.

$$
(E I)_{e q}=E_{f}\left[\frac{b t_{f}^{3}}{6}+\frac{b t_{f} d_{1}^{2}}{2}\right]+E_{i}\left[\frac{b t_{i}^{3}}{6}+\frac{b t_{i} d_{2}^{2}}{2}\right]+E_{c} \frac{b t_{c}^{3}}{12}
$$

As for the case for the ordinary sandwich panel, the contribution of the moment inertia of skin the stiffness of core might be neglected, but the moment inertia of intermediate layer should be taken into account as they have significant thickness. Hence, the above equation, Equation 50, may be reduced to:

$$
(E I)_{e q}=\frac{1}{2} E_{f} b t_{f} d_{1}^{2}+\frac{1}{6} E_{i} b t_{i}^{3}+\frac{1}{2} E_{i} b t_{i} d_{2}^{2}
$$

Following a similar procedure with the analysis for elastic stress distribution in ordinary sandwich beam, the stress at each layer of hybrid sandwich beam can be obtained by replacing the flexural rigidity of ordinary beam with the flexural rigidity of hybrid beam in Equation 51 or Equation 52, which gives:

$$
\sigma_{\mathrm{f}}=\frac{\mathrm{Mz}}{(\mathrm{EI})_{\mathrm{eq}}} \mathrm{E}_{\mathrm{f}}
$$

$$
\begin{gathered}
\text { For } \left.\left.\left[\left(\frac{t_{c}}{2}+t_{i}\right) \leq z \leq\left(\frac{t_{c}}{2}+t_{i}+\frac{t_{i}}{2}\right)\right)\right] ;\left[-\left(\frac{t_{c}}{2}+t_{i}+\frac{t_{i}}{2}\right)\right) \leq z \leq-\left(\frac{t_{c}}{2}+t_{i}\right)\right] \\
\sigma_{i}=\frac{M z}{(E I)_{e q}} E_{i}
\end{gathered}
$$

For $\left[\frac{\mathrm{t}_{\mathrm{c}}}{2} \leq \mathrm{z} \leq\left(\frac{\mathrm{t}_{\mathrm{c}}+\mathrm{t}_{\mathrm{i}}}{2}\right)\right] ;\left[-\left(\frac{\mathrm{t}_{\mathrm{c}}+\mathrm{t}_{\mathrm{i}}}{2}\right) \leq \mathrm{z} \leq-\frac{\mathrm{t}_{\mathrm{c}}}{2}\right]$

$$
\sigma_{\mathrm{c}}=\frac{\mathrm{Mz}}{(\mathrm{EI})_{\mathrm{eq}}} \mathrm{E}_{\mathrm{c}}
$$

For $\left[-\frac{t_{c}}{2} \leq \mathrm{z} \leq \frac{t_{c}}{2}\right]$

In a similar way to the previous analysis, the shear in the core of hybrid sandwich panel can be obtained by also taking into consideration the contribution of intermediate layer.

$$
\begin{aligned}
& \sum A_{c} z_{c} E=E_{f} \cdot A_{f} \cdot \frac{d_{1}}{2}+E_{i} \cdot A_{i} \cdot \frac{d_{2}}{2}+E_{c} \cdot A_{c} \cdot\left[\frac{1}{2}\left(\frac{t_{c}}{2}-z\right)+z\right] \\
& \sum A_{c} b_{c} E=E_{f} \cdot \frac{b t_{f} d_{1}}{2}+E_{i} \cdot \frac{b t_{i} d_{2}}{2}+\frac{E_{c} \cdot b}{2}\left(\frac{t_{c}^{2}}{4}-z^{2}\right)
\end{aligned}
$$


Thus,

$$
\tau=\frac{Q}{b(E I)_{e q}}\left[\frac{E_{f} b_{f} d_{1}}{2}+\frac{E_{i} b t_{i} d_{2}}{2}+\frac{E_{c} \cdot b}{2}\left(\frac{t_{c}^{2}}{4}-z^{2}\right)\right]
$$

By removing the factor of $b$, the final equation can be obtained as follows:

$$
\tau=\frac{Q}{(E I)_{\text {eq }}}\left[\frac{E_{\mathrm{f}} \mathrm{t}_{\mathrm{f}} \mathrm{d}_{1}}{2}+\frac{E_{\mathrm{i}} \mathrm{t}_{\mathrm{i}} \mathrm{d}_{2}}{2}+\frac{\mathrm{E}_{\mathrm{c}}}{2}\left(\frac{\mathrm{t}_{\mathrm{c}}^{2}}{4}-\mathrm{z}^{2}\right)\right]
$$

Finally, the deflection and stiffness of hybrid sandwich panel can be obtained in the similar way with the equations for ordinary sandwich panel. The deflection is the sum of deflection due to bending load and shear of the core, and subsequently the stiffness is the load divided by this deflection. By this process, the analogous equations below for the deflection and stiffness of ordinary beam are proposed for the hybrid sandwich panel with the value of flexural rigidities(EI) $)_{\text {eq }}$ defined by Equation 51 .

$$
\begin{gathered}
\delta=\frac{\mathrm{FL}^{3}}{48(\mathrm{EI})_{\mathrm{eq}}}+\frac{\mathrm{FL}}{4(\mathrm{AG})_{\mathrm{eq}}} \\
\mathrm{k}=\frac{48(\mathrm{EI})_{\mathrm{eq}}}{\mathrm{L}^{3}}+\frac{4(\mathrm{AG})_{\mathrm{eq}}}{\mathrm{L}}
\end{gathered}
$$

\section{CONCLUSIONS}

The theoretical frameworks developed in this study shows that the incorporation of intermediate layer within a sandwich structure can besignificantly enhanced the flexural rigidity and stiffness of the sandwich panel. The equivalent flexural rigidity (EI) eq of hybrid sandwich panel includes the rigidities of the faces, intermediate layer and core measured about the neutral axis of the entirely sections. As a result, the new developed hybrid sandwich panel generates a higher stiffness by only slightly increase in weight contributed by intermediate layer. It is worth noting that the material for intermediate layer should have intermediate properties between the skins and core.

\section{REFERENCES}

Allen, H.C. (1969), Anaysis and Design of Structural Sandwich Panels, Pergamon Press, Oxford.

Deshpande, V.S. (2002), The design of sandwich panels with foam cores, Lecturing Handout, Cambridge University.

DIAB (2001), Sandwich concept, DIAB sandwich book, Available from: www.diabgroup.com.

Fajrin, J., Zhuge, Y., Bullen, F., Wang, H. (2011), The Implementation of Statistical Inference to Study the Bending Strength of Sustainable Hybrid Sandwich Panel, Advanced Material Research, Vol. 250253 (2011), pp. 956-961.

Fajrin, J., Zhuge, Y., Bullen, F., Wang, H. (2011),Flexural Strength of Sandwich Panel with Lignocellulosic Composites Intermediate Layer-aStatistic Approach,International Journal of Protective Structures, Vol 2 (4), pp. 453-464.

Fajrin, J., Zhuge, Y., Bullen, F., Wang, H. (2013), Significance Analysis of Flexural Behaviour of Hybrid Sandwich Panels, Open Journal of Civil Engineering, Vol 3 (2013), pp. 1-7.

Mamalis, A.G., Spentzas, K.N., Pantelelis, N.G., Manolakos, D.E., Ionnidis, M.B. (2008), A New Hybrid Concept for Sandwich Structures, Composite Structures, Vol 83 (2008), pp. 335-340.

Zenkert, D.(1995), An introduction to sandwich construction, Solihull, EMAS. 